\title{
Adjustment Delay Scheme to Improve Performance IEEE 802.15.4 Networks
}

\author{
Eppy Yundra*1, Bih-Hwang Lee ${ }^{2}$ \\ ${ }^{1}$ Universitas Negeri Surabaya, Kampus Ketintang, Surabaya, East Java, Indonesia \\ ${ }^{2}$ National Taiwan University of Science and Technology, \\ 43, Keelung Rd., Section 4, Taipei 106, Taiwan \\ *Corresponding author, e-mail: eppyyundra@unesa.ac.id', bhlee@mail.ntust.edu.tw²
}

\begin{abstract}
The challenges of CSMA/CA as following: first, when the device nodes detect the channel in busy condition, the device nodes have to increase the value of backoff exponent which cause range of blind backoff process also increase. Second, the blind backoff process will cause lower channel utilization and more energy consumptions. This article proposes a scheme to improve IEEE 802.15.4 medium access control, called adjustment delay scheme (ADES). This article also presents a comprehensive Markov chain analysis to predict the probability of successful transmission, network goodput, bandwidth utilization and total network energy consumption. The validity of the model analysis is proven by precisely matching the simulation experiments. ADES performs better than those of other algorithms in term of the probability of successful packet transmission, network goodput, bandwidth utilization as well as energy consumption in the networks.
\end{abstract}

Keywords: IEEE 802.15.4, Markov chain, blind backoff, energy consumption

Copyright $@ 2017$ Universitas Ahmad Dahlan. All rights reserved.

\section{Introduction}

The IEEE 802.15.4 is one of candidates for wireless sensor networks. Wireless sensor networks consist of large number of sensors that are densely deployed to some physical phenomena for wide variety applications. The IEEE 802.15.4 standard has been designed to stipulate the physical sublayer (PHY) and medium access control (MAC) sublayer for low power consumption, short transmission range, and low-rate wireless personal area network (LRWPAN) [1].

Several problems of CSMA/CA as following: first, when the device nodes detect the channel in busy condition, the device nodes have to increase the value of backoff exponent (BE). However, which cause range of blind backoff process also increase. Second, when the device nodes detect the channel in busy condition, the device nodes have to increase number of backoff stage. However, which cause more energy consumptions for enter upcoming backoff stage. In addition, the blind of backoff process in the slotted carrier sense multiple access with collision avoidance (CSMA/CA) will lead to reduce channel utilization and more energy consumptions in the networks.

Analysis of IEEE 802.15.4 MAC performance is still include category of the interest research topics in wireless sensor network. A. N. Alvi at al [2] present an evaluation of the slotted CSMA/CA of IEEE 802.15.4 based on all of its frequency, which only analyze each frequency and compare with each other but not to propose a method. K. Ashrafuzzaman at al [3], the authors use node state and channel state models to analyze the performance of IEEE 802.15.4 MAC that are simple but accurate. The authors also present an analytical in the contention access period (CAP) of the beacon-enabled mode in IEEE 802.15.4 MAC based on model the slotted CSMA/CA algorithm, which only considers for the saturated mode. However, they do not consider about acknowledgement (ACK).

There are several mathematical models for analysis have been presented to analyze the performance of IEEE 802.15.4 based on Markov chain models, but they do not consider packet retransmissions [4-10]. T.-R Park at al [4] have proposed an analyse throughput and energy consumption in saturation conditions based on new markov chain model of 802.15.4 standard. T.-J Lee at al [5] have proposed an evaluate throughput limit in order to grasp the 
characteristics of IEEE 802.15.4 WPAN for a new model based on the slotted CSMA/CA of IEEE 802.15.4 Medium Access Control. S. Pollin at al [6] have proposed an analytical for uplink and acknowledged uplink traffic also evaluation its performance based on star topology network, which are scenarios in both saturated and unsaturated periodic traffic. Y. Zhang at al [7] have studied an optimization packet size which take into considering of the CSMA-CA contention, protocol overhead, and channel condition for IEEE 802.15.4 networks. The authors also have proposed new analytical models to calculate the goodput and the energy consumption. J. He at al [8] have studied an analytical model to estimate the slotted CSMA/CA algorithm detailed in the MAC layer of IEEE 802.15.4 standard based on Markov chain. Z. Xiao at al [9] have presented an analytical performance to IEEE 802.15.4 Medium Access Control (MAC) based on queuing model to provide a tool especially with sleep mode enabled. C. Buratti at al [10] have presented an analytical for the beacon enabled mode of the IEEE 802.15.4 medium-access control (MAC) based on a mathematical model. The multiple nodes are arranged to be a personal area network (PAN), which transmit data pass through directly links or multiple hops to PAN coordinator. Some of the modified Markov chain models have been investigated by considering packet retransmissions but not considering the defer transmission [11-14]. Z. Tao at al [11] have proposed precisely seize all the essential features of the IEEE 802.15.4 MAC based on a novel Markov chain, and thus can provide strengths and weaknesses that valuable concept of multiple access scheme. P. Park at al [12] have studied of reliability, delay, energy consumption and proposed a generalized analysis of the IEEE 802.15.4 medium access control (MAC). Y-K Huang at al [13] have studied duty-cycle operation for comprehensively analyzes IEEE 802.15.4. Specifically, a novel analytical model that accommodates a general traffic distribution is developed. M. Khanafer at al [14] have been proposed method to achieve efficient power savings for the sensor nodes with modification the IEEE 802.15.4 standard in order to improve reliability and provided better channel utilization. In [15-19], the authors propose the Markov chain models with considering the postpone transmission. B. Gao at al [15] have presented an analytical of slotted carrier sense multiple access/collision avoidance (CSMA/CA) algorithm with take into considering the newly enabled sleep mode based on extended Markov for IEEE 802.15.4. C.-Y Jung at al [16] have been presented analytical with reflect of feature the IEEE 802.15.4 medium-access control (MAC) protocol in term of a superframe duration, acknowledgements, and retransmissions with and without limit based on Markov chain. B. Shrestha at al [17] have presented an analytical slotted CSMA/CA for the IEEE 802.15.4 networks considering GTS transmission phenomena together in heterogeneous traffic in term of non-saturation based on general discrete-time Markov chain. B.-H. Lee at al [18] have presented an analytical with determine the precise values of beacon order and superframe order for the PAN coordinator, cluster coordinators, and device nodes, and determine the accurate time for the beacon transmission of PAN and coordinator nodes, respectively. M. Martalo at al [19] have been proposed the analytical model based on Markov chain for multi-hop cluster network has been studied without considering ACK to confirm the successful of data packet transmission. However, all of the abovementioned models only consider for CSMA/CA standard, i.e., not proposed new contention mechanism in CAP

$\mathrm{H} .-\mathrm{K} \mathrm{Wu}$ at al [20] have alse presented an analytical a cluster tree network that is extended with considering acknowledgement, packet retransmission, and defer transmission based on Markov chain model. B.-H. Lee at al [21] also proposed a scheme to improve IEEE 802.15.4 medium access control, called superframe duration adjustment scheme (SUDAS), which analyzes the overall of the IEEE 802.15.4 not only CAP but also CFP. SUDAS is expected to effectively allocate guaranteed time slot to the requested devices, it adjusts the length of the slot in superframe duration based on the length of the packet data.

Several authors have been proposed hybrid method MAC protocol combining CSMA with time division multiple access (TDMA) to rectify the slotted CSMA/CA for wireless sensor network [22-24]. K. Sarvakar at al [22] have been proposed a streamlined hybrid MAC layer with exploit ZigBee MAC (EZ-MAC), for sensor networks. Sensor in the networks have to manage variety of traffics such as real-time traffics and sensor traffics. $\mathrm{H}$. Deng at al [23] have been investigated performance models for CSMA/CA and TDMA systems and compare their performance under both non-saturation and saturation conditions based on the developed performance models. I. Rhee at al [24] have proposed a hybrid MAC protocol such as the design, implementation and achievement evaluation with integrating strengths of TDMA and CSMA while off setting their weaknesses called Z-MAC for wireless sensor networks.

Adjustment Delay Scheme to Improve Performance IEEE 802.15.4 Networks (Eppy Yundra) 
R. K Patro at al [25] also presented the analysis correctly for contention access period, but they do not consider about defer transmission if the current superframe is not enough to accommodate transmission. M.B Rasheed at al [26] have proposed an analysis for slotted CSMA/CA for energy consumption, but they only consider about idle condition for the standard and do not propose a new scheme. B-H Lee at al [27] have presented an analytical invorder to improvement performance star networks by using extra carrier sensing for IEEE 802.15.4. However, the authors do not consider about defer transmission and they do not provide retransmission state in Markov chain model. In [29-30], sensor nodes use different mechanisms to reduce the waiting time before they have successfully transmitted packets, which makes it possible to decrease the backoff time between packet transmissions. Ji. $Z$ at al [28] have presented an extended for personal area networks in many area industrial applications such as E-Healthcare, environmental monitoring and industrial automation with few complication and major reliability which enable PANs to be greatly applied. Lu ZQ at al [29] have studied an manage connectivity and detecting nodes or link failures is difficult in unstructured WSN because there is large number of nodes. The sensor nodes are tiny devices with limited constraints and it is not feasible in deployed scenarios to recharging the batteries. Therefore to decrease energy consumption and prolonging the WSN lifetime is main design objective for sensor based applications and mechanisms. B. Bougard at al [30] have studied an apply ultra low power sensor node with exploit potency of an 802.15.4 radio for implementation in a dense network area. C.M Wong at al [31] have proposed also using extra adding carrier sensing algorithm based on IEEE 802.15.4 to increase performance in authentic slotted CSMA/CA. However, fail to alleviate collisions.

This article proposes an adjustment delay scheme (ADES) for IEEE 802.15.4 which is the extended work from [18] and [21]. In [18] the authors focus on how to regulate the beacon starting times of personal area network and coordinator nodes in order to decrease the collisions between beacons or even between beacon and data packets for cluster tree topology. In [21], the authors focus how to determine the accurate time for the GTS starting time (GTSstart) and the GTS length (GTSlength) on setting adaptable length of GTS slot based on the length of packet for star topology. However, all of the aforementioned models only consider CSMA/CA standard for contention mechanism. In other words, they did not consider the blind of backoff and probability going to upcoming backoff stage. ADES focuses on adjustment delay if device node detects the channel in busy condition which can reduce probability of going to entering upcoming backoff stage and blind of backoff process. In addition, this scheme also include addition third CCA for reduce collision. The performance of ADES for star topology is analyzed by the Markov chain model modified from [18] for obtain the probability of success transmissions, network goodput, bandwidth utilization as well as energy consumption which considering packet retransmission, ACK and defer transmission for IEEE 802.15.4 networks. The major contribution of this article is to model the channel access for star network that analyze overall of performance IEEE 802.15 .4 to minimize of probability going to upcoming backoff stage which can increase probability success packet transmission with adjustment delay at first CCA and second CCA if channel find in busy condition also including addition third CCA to reduce collision in order to improve performance networks.

\section{Research Method}

\subsection{The Description of ADES}

ADES is expected to adjust delay if the device node detects the channel in busy condition. If the device node detects channel in busy condition which cause blind of backoff process. In this case can cause degradation of performance networks not only lower bandwidth utilization but also more energy consumption. Let us denote $S D$ and $T_{\text {delay }}$ be the supeframe duration in second and the time interval of waiting due to CCA busy in second, which can be obtained by equations (1) and (2), respectively, where aBaseSuperframeDuration is the minimum duration of the superframe equal to 960 symbols and $R s$ is denote symbol data rate equal to $62500 \mathrm{symbol} / \mathrm{second}$.

$$
S D=\frac{\text { aBaseSuperframeDuration } \times 2^{S O}}{R S} \text { [in second] }
$$

TELKOMNIKA Vol. 15, No. 4, December 2017: 1710 - 1722 


$$
T_{\text {delay }}=\frac{1 \times \text { Unitbackgferiod }}{R \mathrm{~s}}[\text { in second] }
$$

According to IEEE 802.15.4 standard for format of the superframe structure is can be shown in Figure 1. Furthermore, the part of active portion of each superframe consist of three arrangements such as beacon, CAP and CFP, which is started 0 to 15 equal length slots. The length of one slot is equal to aBaseSlotDuration $\times 2^{\text {so }}$ symbols, where aBaseSlotDuration is equal to 60 symbols.

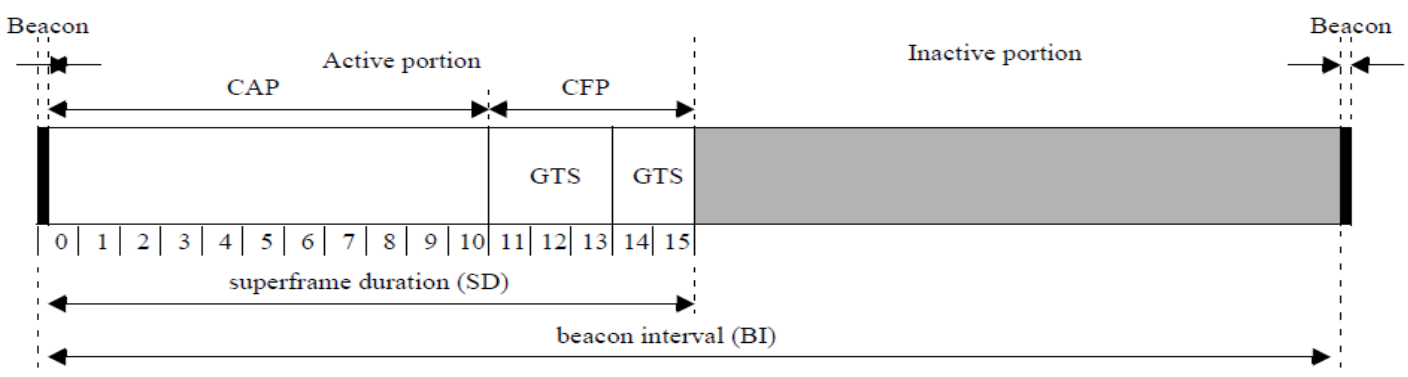

Figure 1. An example of superframe structure

In this CAP, each node will communicate using the slotted CSMA/CA in CAP with the network coordinator. In order to transmit a packet, first each node will delay a backoff period (BP) decided by randomly choosing from 0 to $\left(2^{\mathrm{BE}}\right.$ minus 1$)$ UBP, where $\mathrm{BE}$ is a backoff exponent and at the beginning set to the value of aMinBE, and then it will performs the first CCA to detect channel condition. If the channel condition is detected in busy at the first CCA detection, it will reduce minus one the value of contention window (CW), then it will continue delay for one slot $T_{\text {delay }}$ and then it will performs again the second CCA to detect the channel condition. If the second CCA channel is detected by busy condition, the node will reduce again with minus one for value of contention window (CW), then continue delay for two slot $T_{\text {delay }}$ and then performs again for third CCA If the third CCA channel is detected by idle condition, the node will try to start transmitting data packet and waits for the acknowledgment packet from the coordinator until finishing transmission. However, if third CCA channel is detected by busy condition, the device node will do it again a backoff period (BP) between 0 and $\left(2^{(B E+1)}\right.$ minus 1) UBP and will to try CCA again, where value of BE can be achieved to the maximum of value macMaxBE. The transmission of system will be failed if the total number aggregation of backoff attempts (NB) outpace the value macMaxCSMABackoffs. For more detail about the aforementioned description, we can explain ADES with flowchart as shown in Figure 2. We take into consideration a topology use star network which consist of one network coordinator and surrounded several device nodes. By using Equation (2), we can get the value of $T_{\text {delay }}$ for each CCA when channel in busy condition.

Basically, the flowchart of the ADES algorithm can be obtained by somewhat modified the IEEE 802.15.4 standard, which can be shown in Figure 2. While Markov chain model that as shown in Figure 5. The first step set the contention window (CW) to 3 for make sure there are three CCAs and there are two conditional judgment for verify of CCA1 and CCA2 detection. If $\mathrm{CW}$ is equal to three after detecting a busy conditionl, certainly CCA1 failed. If the value of CW is equal to two after detecting busy channel, certainly CCA2 also failed. The system will continue to carry out CCA3 after postpone two of slot durations of $T_{\text {delay }}$. If verify of CCA3 the channel in busy again, so the device node has to go to the upcoming backoff stage. An example comparation of ADES with IEEE 802.15.4 standard can be shown in Figures 3 and 4, respectively. The ADES scheme have the probability going to upcoming backoff stage less than IEEE 802.15.4 standard, so that the ADES scheme can improve the network performance. 


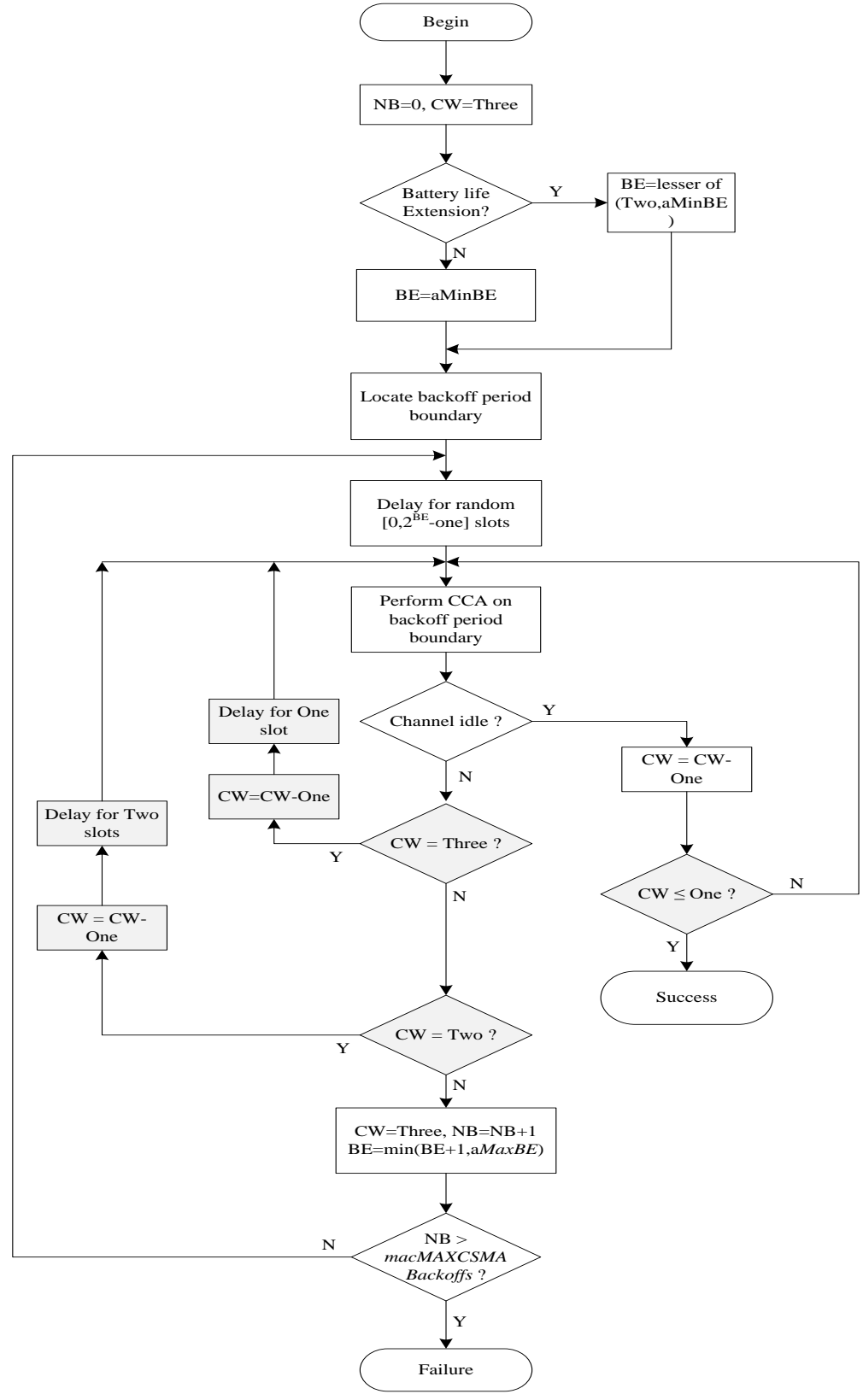

Figure 2. The flowchart of ADES

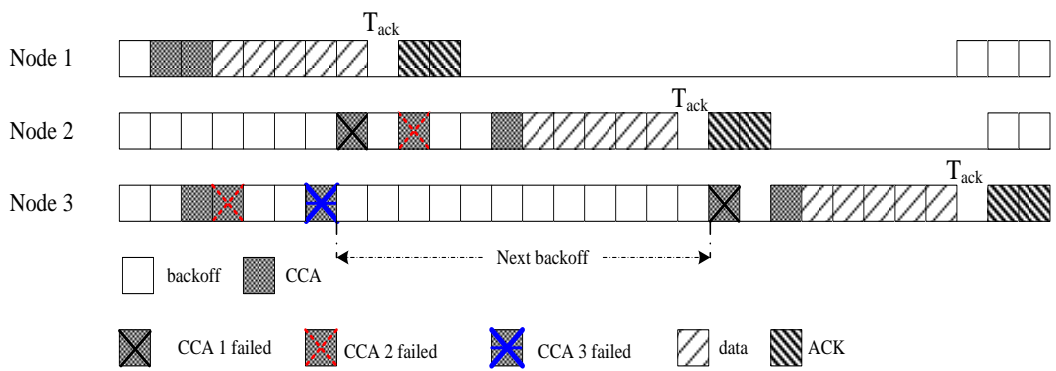

Figure 3. An example of implementation of ADES 


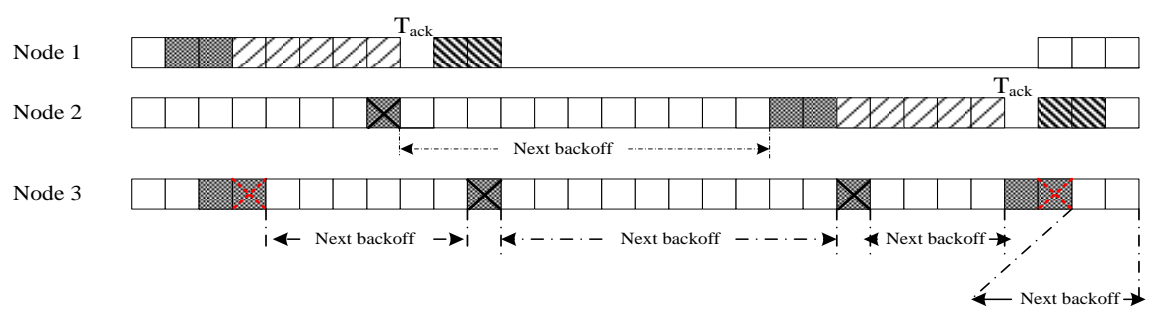

$\square$ backoff $\mathrm{CCA}_{\mathrm{CCA} 1 \text { failed }} \mathrm{CCA}_{2 \text { failed }} \mathrm{CCA} 3$ failed $^{\mathrm{C}} \mathrm{data}^{\mathrm{ACK}}$

Figure 4. An example of implementation of IEEE 802.15.4

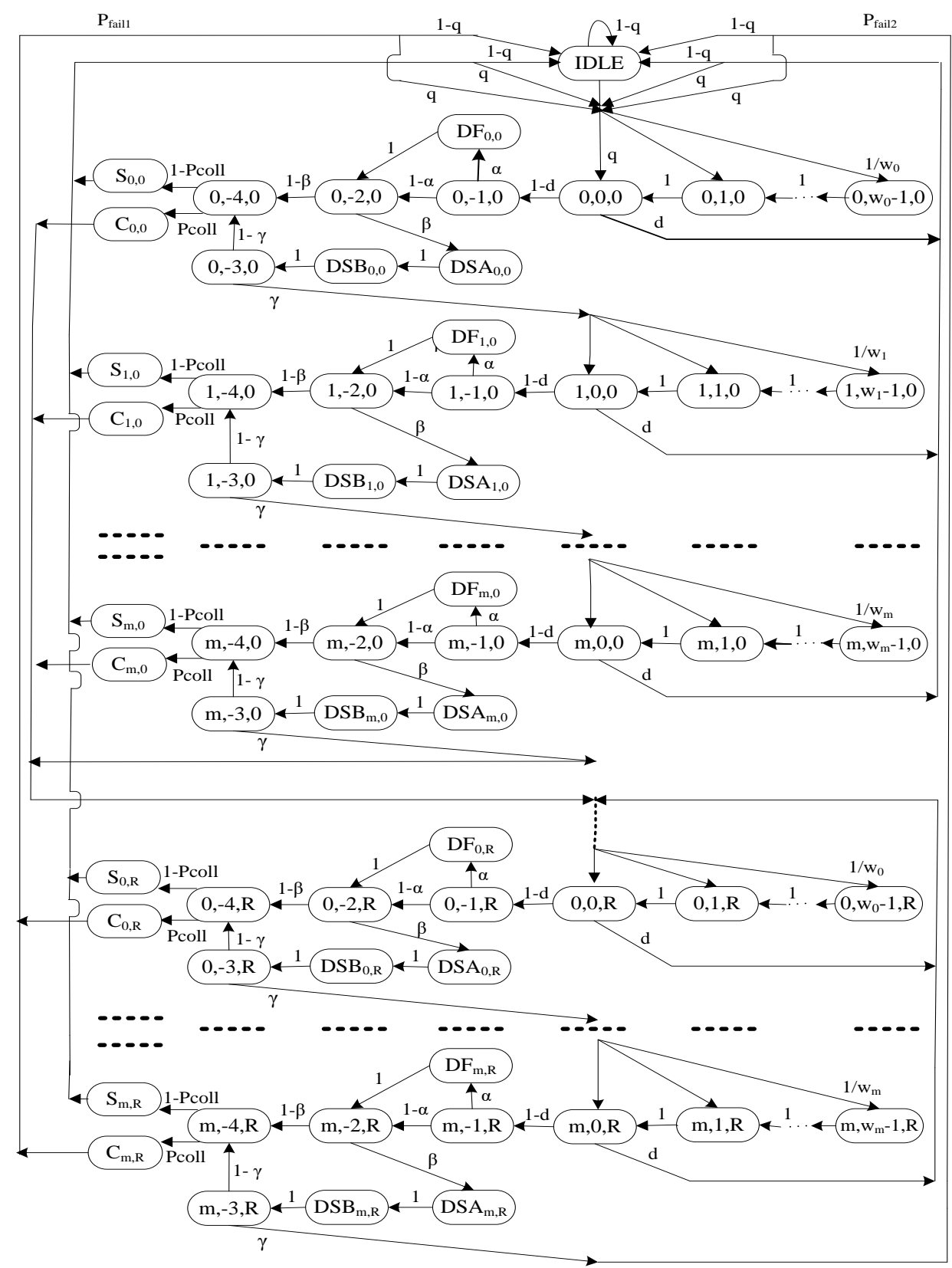

Figure 5. Markov chain model for ADES 


\subsection{Analysis of ADES}

In this subsection, the proposed ADES based on the IEEE 802.15.4 by using CSMA/CA for active portion, part of CAP only, because we only consider about active period, while part of CFP is neglected. This article also take into considering the case of acknowledged uplink data transmission is investigated comprehensively by Markov chain model as shown in figure 5 .

Let bi,j,k be the stationary probability at the stochastic state $(\mathrm{s}(\mathrm{t})=\mathrm{i}, \mathrm{c}(\mathrm{t})=\mathrm{j}$, and $\mathrm{r}(\mathrm{t})=$ $k$ ), where $s(t), c(t)$, and $r(t)$ reflect backoff stage, backoff counter, and number of retransmissions, respectively, shown as Equation (4), where $b_{i,-1, k}, b_{i,-2, k}, b_{i,-3, k}$ and $b_{i,-4, k}$ are the stationary probabilities for the first CCA $\left(\mathrm{CCA}_{1}\right)$, the second CCA $\left(\mathrm{CCA}_{2}\right)$, the third CCA (CCA3) and packet transmission, respectively, at the $\mathrm{i}^{\text {th }}$ backoff stage and the $\mathrm{k}^{\mathrm{th}}$ retransmission. Let $\mathrm{b}_{\mathrm{si}, \mathrm{k}}$ and $b_{\mathrm{C}, \mathrm{k}}$ be the stationary probabilities of the successful transmission and collision at the states of $S_{i, k}$ and $C_{i, k}$ as shown in Equations (5) and (6), respectively, where $m$ and $R$ are the maximum NB stage and retransmissions, i.e., they are equal to 4 and 3 , respectively. Let $b_{D F i, k}, b_{D S A i, k}$ and $b_{D S B i, k}$ be the stationary probabilities of delay one slot at first CCA busy, delay one slot for second CCA busy for first and delay one slot for second CCA busy for second at the states of $D_{i, k}, D_{S A}$, and $D_{i, k}, k$ for the $i^{\text {th }}$ backoff stage and the $k^{\text {th }}$ retransmission as shown in equations. (7) to (9), respectively.

$$
\begin{aligned}
& \mathrm{b}_{\mathrm{i}, \mathrm{j}, \mathrm{k}}=\lim _{\mathrm{t} \rightarrow \infty} \mathrm{P}\{\mathrm{s}(\mathrm{t})=\mathrm{i}, \mathrm{c}(\mathrm{t})=\mathrm{j}, \mathrm{r}(\mathrm{t})=\mathrm{k}\}, \text { for } \mathrm{i} \in(0, \mathrm{~m}), \mathrm{j} \in\left(-4, \mathrm{w}_{\mathrm{i}}-1\right), \mathrm{k} \in(0, \mathrm{R}) \\
& \mathrm{b}_{\mathrm{s}_{\mathrm{i}, \mathrm{k}}}=\lim _{\mathrm{t} \rightarrow \infty} \mathrm{P}\left\{\mathrm{S}_{\mathrm{s}(\mathrm{t})}=\mathrm{S}_{\mathrm{i}}, \mathrm{r}(\mathrm{t})=\mathrm{k}\right\}, \mathrm{i} \in(0, \mathrm{~m}), \mathrm{k} \in(0, \mathrm{R}) \\
& \mathrm{b}_{\mathrm{C}_{\mathrm{i}, \mathrm{k}}}=\lim _{\mathrm{t} \rightarrow \infty} \mathrm{P}\left\{\mathrm{C}_{\mathrm{s}(\mathrm{t})}=\mathrm{C}_{\mathrm{i}}, \mathrm{r}(\mathrm{t})=\mathrm{k}\right\}, \mathrm{i} \in(0, \mathrm{~m}), \mathrm{k} \in(0, \mathrm{R}) \\
& \mathrm{b}_{\mathrm{DF}_{\mathrm{i}, \mathrm{k}}}=\lim _{\mathrm{t} \rightarrow \infty} \mathrm{P}\left\{\mathrm{DF}_{\mathrm{s}(\mathrm{t})}=\mathrm{DF}_{\mathrm{i}}, \mathrm{r}(\mathrm{t})=\mathrm{k}\right\}, \mathrm{i} \in(0, \mathrm{~m}), \mathrm{k} \in(0, \mathrm{R}) \\
& \mathrm{b}_{\mathrm{DSA}_{\mathrm{i}, \mathrm{k}}}=\lim _{\mathrm{t} \rightarrow \infty} \mathrm{P}\left\{\mathrm{DSA}_{\mathrm{s}(\mathrm{t})}=\mathrm{DSA}_{\mathrm{i}}, \mathrm{r}(\mathrm{t})=\mathrm{k}\right\}, \mathrm{i} \in(0, \mathrm{~m}), \mathrm{k} \in(0, \mathrm{R}) \\
& \mathrm{b}_{\mathrm{DSB}_{, \mathrm{k}}}=\lim _{\mathrm{t} \rightarrow \infty} \mathrm{P}\left\{\mathrm{DSB}_{\mathrm{s}(\mathrm{t})}=\mathrm{DSB}_{\mathrm{i}}, \mathrm{r}(\mathrm{t})=\mathrm{k}\right\}, \mathrm{i} \in(0, \mathrm{~m}), \mathrm{k} \in(0, \mathrm{R})
\end{aligned}
$$

Let us explain the parameters used in the Markov chain model as follows. Let $w_{i}=2^{B E_{i}}$ be the backoff window at the $i^{\text {th }}$ backoff stage of a device, where the backoff exponent $B E_{i}=3$, $4,5,5$, and 5 for $0 \leq \mathrm{i} \leq \mathrm{m}$. An IDLE state means that a device node has no packet to transmit. Let denote $q$ be the probability a packet come at a node during the active period.

In this CSMA, if the remaining slot, i.e., CCA analysis, the frame transmission, and any ACK can be completed before the end of $C A P_{\text {ades }}$, then the MAC sublayer should transmit its packet. Conversely, if the current $\mathrm{CAP}_{\text {ades }}$ has not enough slots to transmit data packets, it should defer transmission until the beginning of the $\mathrm{CAP}_{\text {ades }}$ in the upcoming superframe duration. Let $d$ be the probability of defer transmission that no enough slot is left in the current $\mathrm{CAP}_{\text {ades }}$ to transmit data packet, which can be obtained by equation (10), where $T_{\text {txCCA, }}, T_{\text {data, }}, T_{\text {ack }}$, $T_{\text {Lack, }} T_{\text {LIFS, }}$ are the time to transmit CCA, time to transmit data packet, time to waiting for ACK packet, time to transmit ACK packet and time duration of IFS, respectively. The value of $L_{\text {ack }}$ is equal to 11 bytes, while the value of $L_{\text {IFS }}$ is equal to macMinLIFSPeriod (20 bytes) if the value of packet is greater than aMaxSIFSFrameSize (18 bytes), otherwise, it is equal to macMinSIFSPeriod ( 6 bytes).

$$
\mathrm{d}=\frac{3 \mathrm{~T}_{\text {txCCA }}+3 \mathrm{~T}_{\text {delay }}+\mathrm{T}_{\text {data }}+\mathrm{T}_{\text {ack }}+\mathrm{T}_{\text {Lack }}+T_{\text {LIFS }}}{\mathrm{CAP}_{\text {ades }}}
$$


Let us denote $\alpha, \beta$ and $y$ be the probabilities that $\mathrm{CCA}_{1}, \mathrm{CCA}_{2}$ and $\mathrm{CCA}_{3}$ are busy, respectively. $C C A_{1}$ busy that means the device node at one of the $C C A_{1}$ states while at least one of the other nodes at during packet transmission state, while $\mathrm{CCA}_{2}$ busy that means the device node at one of the $\mathrm{CCA}_{2}$ states while at least one of the other nodes at during packet transmission state and $\mathrm{CCA}_{3}$ busy that means the device node at one of the $\mathrm{CCA}_{3}$ states while at least one of the other nodes at during packet transmission state. Let us also denote $P_{\text {coll }}$ to be the probability of the collision transmission of packet after $\mathrm{CCA}_{2}$ or CCA3, i.e., the device node at packet transmission state while at least one of the other nodes in during the packet transmission state at the same time, which can be obtained by equation (11). Let us also denote $P_{\text {fail } 1}$ and $P_{\text {fail } 2}$ to be the probabilities of fail transmission due to the maximum number of retransmissions after collisions and no channel to use after reaching the maximum backoff stage at the maximum retransmission stage, as shown in Equations (12) to (13), respectively.

$$
\begin{aligned}
& P_{\text {coll }}=N \tau\left(1-(1-\tau)^{(N-1)}\right) \\
& P_{\text {fail } 1}=\sum_{\mathrm{i}=0}^{\mathrm{m}} \mathrm{b}_{\mathrm{C}_{\mathrm{i}, \mathrm{R}}}=\mathrm{b}_{0,0,0}\left(\mathrm{X}^{\mathrm{R}}+\mathrm{Z}^{\mathrm{R}}\right) \mathrm{X} \\
& P_{\text {fail } 2}=\mathrm{b}_{\mathrm{m}, 0 \mathrm{R}}(1-d) \beta \gamma=\mathrm{b}_{0,0,0}\left(\mathrm{Y}^{\mathrm{m}}\right)\left(\mathrm{X}^{\mathrm{R}}+\mathrm{Z}^{\mathrm{R}}\right)(1-d) \beta \gamma
\end{aligned}
$$

Let denote $P_{\mathrm{cr}}$ to be the probability of collision transmission after $k$ attempts (Probability of packet being dropped due to collision retransmission), which can be calculated by equation (14). Let $P_{\text {dropcoord }}$ and $P_{\text {succoord }}$ be the probabilities of drop transmission and successful transmission from device node to its coordinator, which can be obtained by Equtions (15) and (16), respectively. Let us also denote $N_{\text {recvcoord }}$ and $T_{\text {sim }}$ to be the number of packets are received by the network coordinator and time of simulation, which can be calculated by equation (17). Therefore the goodput of ADES in the network which denoted by $S_{\text {ades, }}$, can be calculated by Equation (18).

$$
\begin{aligned}
& P_{\text {cr }}=\sum_{\mathrm{k}=1}^{\mathrm{R}}\left(P_{\text {coll }}\right)^{\mathrm{k}} \\
& P_{\text {dropcoord }}=P_{\text {cr }}+P_{\text {fail1 }}+P_{\text {fail } 2} \\
& P_{\text {succoord }}=1-P_{\text {dropcoord }} \\
& N_{\text {recvcoord }}=\frac{\lambda_{\mathrm{n}} \times N \times\left(1-P_{\text {dropcoord }}\right) \times T_{\text {sim }}}{L_{\text {data }}} \\
& S_{\text {ades }}=\frac{N_{\text {recvcoord }} \times \mathrm{L}_{\text {data }}}{T_{\text {sim }}}
\end{aligned}
$$

Let also denote $N \mathrm{Tq}_{1}$ and $N \mathrm{Tq}_{2}$ be number of packets received by coordinator using $\mathrm{TQ}_{1}$ and TQ2, respectively. Let us denote $N_{\text {beacon }}$ and $B U_{\text {total }}$ be number of beacons and bandwidth utilization in the networks, which can be obtained by equations (19) and (20), respectively.

$$
N_{\text {beacon }}=\frac{T_{\text {sim }}}{S D}
$$




$$
B U_{\text {total }}=\frac{\left(N T q_{1} \times T Q_{1}\right)+\left(N T q_{2} \times T Q_{2}\right)}{N_{\text {beacon }} \times C A P_{\text {ades }}}
$$

The energy consumption of device can be shown in Equation (21) and the energy consumption of coordinator node can be shown in Equation (22). Finally, the total energy consumption in networks consist of the energy consumption by devices node and coordinator node can be shown in Equation (23).

$$
\begin{aligned}
& \mathrm{E}_{\text {dev }}=\left(\mathrm{PWR}_{\mathrm{idle}} \sum_{\mathrm{i}=0}^{\mathrm{m}} \sum_{\mathrm{j}=0}^{\mathrm{w}_{\mathrm{i}}-1} \sum_{\mathrm{k}=0}^{\mathrm{R}} \mathrm{b}_{\mathrm{i}, \mathrm{j}, \mathrm{k}} \times \mathrm{SD}_{\text {Coord }} \times \frac{\text { Time }}{\mathrm{BI}_{\text {Coord }}} \times N\right)+ \\
& \left(3 \mathrm{PWR}_{\mathrm{tx}} \frac{\left(\lambda_{n}\right)}{C A P_{\text {ades }}} \times \frac{\mathrm{L}_{\mathrm{cca}}}{\mathrm{R}_{\mathrm{b}}} \sum_{\mathrm{i}=0}^{\mathrm{m}} \sum_{\mathrm{k}=0}^{\mathrm{R}}\left(\mathrm{b}_{\mathrm{i},-1 \mathrm{k}}+\mathrm{b}_{\mathrm{i},-2 \mathrm{k}}+\mathrm{b}_{\mathrm{i},-3 \mathrm{k}}\right) \times \mathrm{T}_{\text {sim }} \times \mathrm{D}_{\text {node }} \times \mathrm{N}\right)+ \\
& \left(\mathrm{PWR}_{\mathrm{tx}} \frac{\left(\lambda_{h}\right)}{C A P_{\text {ades }}} \times \frac{\mathrm{L}_{\text {data }}}{\mathrm{R}_{\mathrm{b}}} \times \mathrm{P}_{\text {succoord }} \times \mathrm{T}_{\text {sim }} \times \mathrm{D}_{\text {node }} \times \mathrm{N}\right)+ \\
& \left(\mathrm{PWR}_{\mathrm{tx}} \frac{\left(\lambda_{n}\right)}{C A P_{\text {ades }}} \times \frac{\mathrm{L}_{\text {delay }}}{\mathrm{R}_{\mathrm{b}}} \times \mathrm{P}_{\text {deconsec }} \times \mathrm{T}_{\text {sim }} \times \mathrm{N}\right)+\left(\mathrm{PWR}_{\text {idle }} \times \mathrm{P}_{\text {idle }} \times \mathrm{SD}_{\text {Coord }} \frac{\mathrm{T}_{\text {sim }}}{\mathrm{BI}_{\text {Coord }}} \times N\right)+ \\
& \left(\mathrm{PWR}_{\mathrm{rx}} \frac{\mathrm{L}_{\text {beacon }}}{\mathrm{R}_{\mathrm{b}}} \times \frac{\mathrm{T}_{\text {sim }}}{\mathrm{BI}_{\text {coord }}} \times N\right)+\left(\mathrm{PWR}_{\mathrm{rx}} \frac{\left(\lambda_{h}\right)}{C A P_{\text {ades }}} \times \frac{\mathrm{L}_{\text {ack }}}{\mathrm{R}_{\mathrm{b}}} \times \mathrm{P}_{\text {succoord }} \mathrm{T}_{\text {sim }} \times \mathrm{N}\right)+ \\
& \left(\mathrm{PWR}_{\mathrm{tx}} \times Y \times \frac{S D_{\text {Coord }}}{B I_{\text {Coord }}} \times T_{\text {sim }} \times N\right) \\
& \mathrm{E}_{\text {Coord }}=\left(\mathrm{PWR}_{\text {idle }} \times \mathrm{P}[\mathrm{IDLE}] \times \mathrm{SD}_{\text {Coord }} \frac{\mathrm{T}_{\text {sim }}}{\mathrm{BI}_{\text {Coord }}}\right)+\left(\mathrm{PWR}_{\mathrm{tx}} \times \frac{\mathrm{L}_{\text {beacon }}}{\mathrm{R}_{\mathrm{b}}} \times \frac{\mathrm{T}_{\text {sim }}}{\mathrm{BI}_{\text {Coord }}} \mathrm{D}_{\text {node }}\right)+ \\
& \left(P W R_{r x} \frac{\left(\lambda_{n}\right)}{C A P} \times \frac{\mathrm{L}_{\text {data }}}{\mathrm{R}_{\mathrm{b}}} \mathrm{P}_{\text {succoord }} \times \mathrm{T}_{\text {sim }} \times \mathrm{N}\right)+ \\
& \left(\mathrm{PWR}_{\mathrm{tx}} \frac{\left(\lambda_{n}\right)}{C A P} \times \frac{\mathrm{L}_{\text {ack }}}{\mathrm{R}_{\mathrm{b}}} \times \mathrm{P}_{\text {succoord }} \times \mathrm{T}_{\text {sim }} \times \mathrm{D}_{\text {node }} \times \mathrm{N}\right) \\
& \mathrm{E}_{\text {total }}=\mathrm{E}_{\mathrm{dev}}+\mathrm{E}_{\text {Coord }}
\end{aligned}
$$

\section{Results and Analysis}

In this section, extended Castalia simulator is used to simulate ADES in order to validate analysis and performance evaluation. The performance of ADES is compared with the SUDAS and the IEEE 802.15.4 standard, which include of the analytical (ana) and simulation (sim) results. We take into considering a topology star network with one PAN coordinator is located on center and 20 device nodes are located its surround, where $D_{\text {node }}$ is equal to 10 meters. To perform simulation the performance of power consumption, we take into considering the radio parameters of Chipcon's CC2420 2.4 GHz for the IEEE 802.15.4 RF transceiver [32], where the power transmitting $P W R_{\mathrm{tx}}$, the power receiving $P W R_{\mathrm{rx}}$, and the power idle $P W R_{\text {idle, }}$, are $31.32 \mathrm{~mW}, 35.28 \mathrm{~mW}$, and $712 \mu \mathrm{W}$, respectively [30]. The BO and SO settings appropriate the IEEE 802.15.4 standard and the proposed ADES algorithm, which are fixed to be six. We compute the probability of successful packet transmission, network goodput, bandwidth 
utilization and total network energy consumption, where traffic load varies from 0.1 to 1 (full loaded). Table 1 summarizes the simulation parameters.

Table 1. The Simulation Parameters

\begin{tabular}{ll}
\hline Parameter & Value \\
\hline Physical data rate & $250 \mathrm{kbps}$ \\
Packet length $\left(\mathrm{L}_{\text {data }}\right)$ & $720 \mathrm{bits}$ \\
UBP & $80 \mathrm{bits}$ \\
NumSuperframeSlots & 16 \\
MacPacketOverhead & $112 \mathrm{bits}$ \\
ACK length $\left(\mathrm{L}_{\text {ack }}\right)$ & $88 \mathrm{bits}$ \\
$\mathrm{D}_{\text {node }}$ & $10 \mathrm{~m}$ \\
$\mathrm{PWR}_{\mathrm{tx}}$ & $31.32 \mathrm{~mW}$ \\
$\mathrm{PWR}_{\mathrm{rx}}$ & $35.28 \mathrm{~mW}$ \\
$\mathrm{PWR}_{\text {idle }}$ & $712 \mu \mathrm{W}$ \\
$\mathrm{BO}_{\mathrm{SO}}$ & 6 \\
$\mathrm{BE}_{\min }$ & 3 \\
$\mathrm{BE}_{\max }$ & 5 \\
\hline
\end{tabular}

Figure 6 shows the probability of successful transmission arriving at the PAN coordinator against the traffic load by analytical and simulation. The proposed ADES algorithm has higher probability of successful transmissions than those of other algorithms, because adjustment delay and addition third CCA can effectively avoid collision in the network. The average probability of successful transmission of ADES increases by $0.14 \%$ and $2.68 \%$ compared to SUDAS and IEEE 802.15.4 standard, respectively.

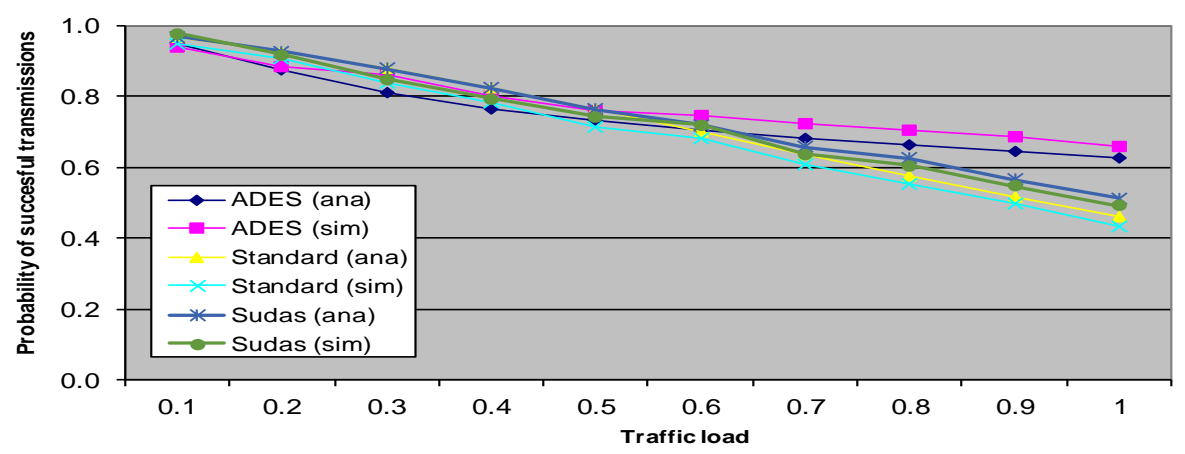

Figure 6. The probability of successful transmission against traffic load

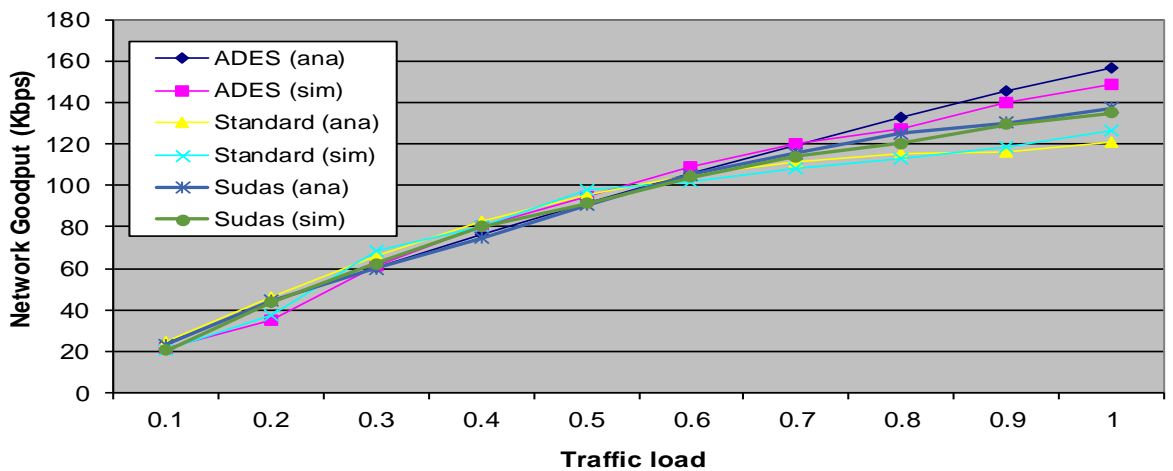

Figure 7. The network goodput against traffic load 
Figure 7 shows the network goodput against traffic load. It is obvious the network goodput of ADES is higher than those of the other algorithms. In the light traffic load (i.e., traffic load is started 0.1 to 0.6 ), the network goodput of ADES is almost the same as those of SUDAS and IEEE standard; however, ADES outperforms to the other algorithms as the traffic load increases (i.e., traffic load is started 0.6 to 1). The average goodput of ADES increases by $5.2 \%$ and $7.6 \%$ compared to SUDAS and IEEE 802.15.4 standard, respectively

Figure 8 shows the bandwidth utilization (BU) against traffic load. The bandwidth utilization of ADES has better efficiency than those of the other algorithms. The average bandwidth utilization of ADES increases by $1.75 \%$ and $5.72 \%$ compared to SUDAS and IEEE 802.15.4 standard, respectively. ADES can improve the bandwidth utilization because reduce probability going to upcoming backoff stage, which cause blind of backoff process.

Figure 9 shows the network energy consumption against traffic load. The average energy consumption of ADES reduces by $18.2 \%$ and $24.2 \%$ compared to SUDAS and IEEE standard, respectively. ADES consumes lesser network energy than those of the other algorithms, because the ADES algorithm can adjust the delay when CCA in busy condition which not only reduce probability collision but also probability entering upcoming backoff stage. Moreover, ADES has greater probability of successful transmission than those of the other algorithms, especially in heavy traffic load, which means that ADES minimizes the energy consumption when retransmitting data packet. The energy consumption is obtained by summing the energy consumption of PAN coordinator and all of device nodes in the network.

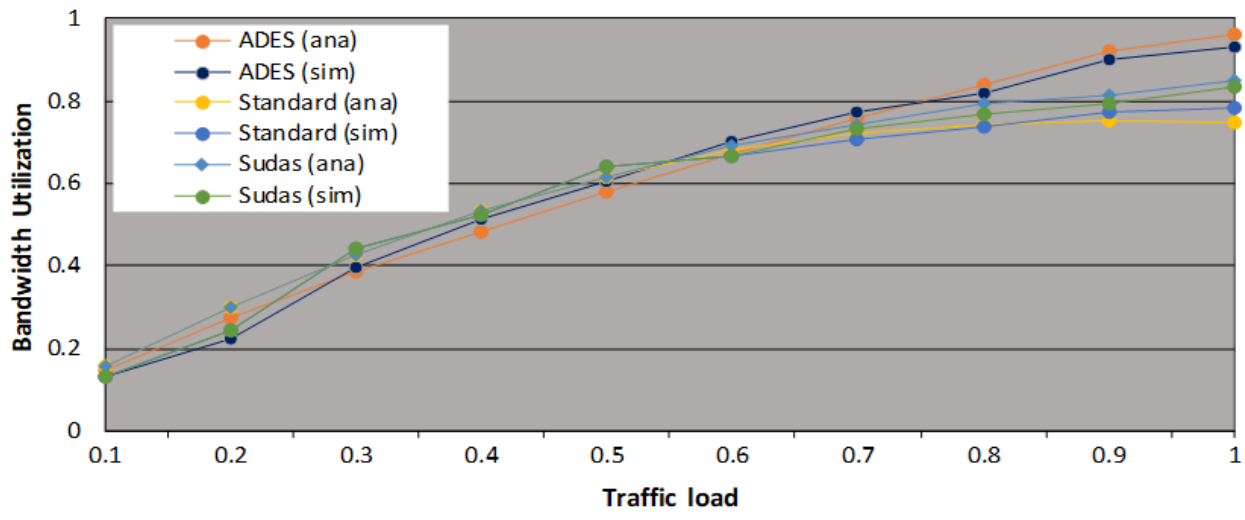

Figure 8. The bandwidth utilization against traffic load

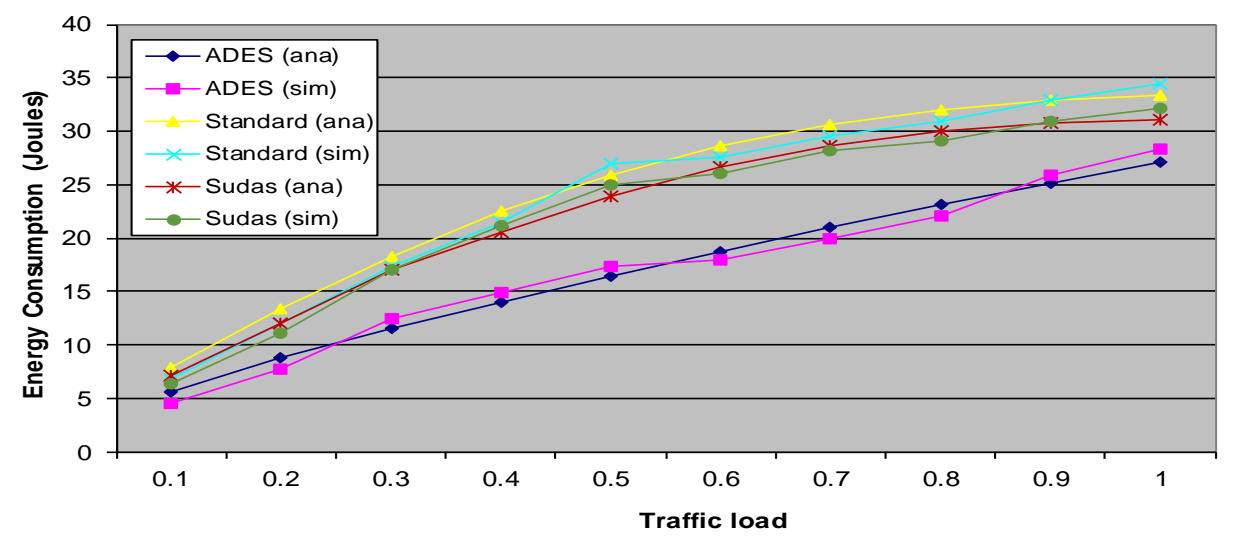

Figure 9. The total of network energy consumptions against traffic load 


\section{Conclusion}

In this article, ADES is presented to improve performance IEEE 802.15.4 networks in order to decrease collision and blind of backoff process which need more energy consumption for random backoff. The proposed ADES algorithm can adjust delay effectively for CCA when channel find in busy condition. Adjustment delay and addition third CCA not only reduce probability collision but also reduce probability going to upcoming backoff stage.

This article also included a comprehensive Markov chain analysis of IEEE 802.15.4, especially for topology star network, to predict the probability of successful transmission, the network goodput, bandwidth utilization as well as the network energy consumption. The validity of the analytical model is shown by precisely matching its predictions of the simulation results. The results of analytical model and simulation experiment show that the performance of ADES is better than those of the other algorithms in term of the probability of successful transmission, network goodput, bandwidth utilization as well as energy consumption.

\section{References}

[1] IEEE 802.15.4. Part 15.4: Wireless Medium Access Control (MAC) And Physical Layer (PHY) Specifications for Low-Rate Wireless Personal Area Networks (Wpans). IEEE standard for information technology. 2006; 15(4).

[2] AN Alvi, SS Naqvi, SH Bouk, N Javaid, U Qasim, ZA Khan. Evaluation of Slotted CSMA/CA of IEEE 802.15.4. Seventh International Conference on Broadband, Wireless Computing, Communication and Applications. Canada. 2012.

[3] K Ashrafuzzaman, K Sup Kwak. On the Performance Analysis of the Contention Access Period of EEE 802.15.4 MAC. IEEE Communications Letters. 2011; 15 (9).

[4] TR Park, TH Kim, JY Choi, S Choi, WH Kwon. Throughput and energy consumption analysis of IEEE 802.15.4 slotted CSMA/CA. IEEE Electronics Letters. 2005; 18(41): 1017-1019.

[5] TJ Lee, HR Lee, MY Chung. MAC throughput limits analysis of slotted CSMA/CA in IEEE 802.15.4 WPAN. IEEE Communications Letters. 2006; 10(7): 561- 563.

[6] S Pollin, M Ergen, S Ergen, B Bougard, L Der Perre, I Moerman, A Bahai, P Varaiya, F Catthoor. Performance Analysis of Slotted Carrier Sense IEEE 802.15.4 Medium Access Layer. IEEE Transactions on Wireless Communications. 2008; 7(9): 3359-3371.

[7] Y Zhang, F Shu. Packet Size Optimization for Goodput and Energy Efficiency Enhancement in Slotted IEEE 802.15.4 Networks. IEEE Wireless Communications and Networking Conference. 2009: $1-6$.

[8] J He, Z Tang, HH Chen, Q Zhang. An accurate and scalable analytical model for IEEE 802.15.4 slotted CSMA/CA networks. IEEE Transactions on Wireless Communications. 2009; 8(1): 440-448.

[9] Z Xiao, C He, L Jiang. An analytical model for IEEE 802.15.4 with sleep mode based on time-varying queue. IEEE International Conference on Communications (ICC), Kyoto, Japan. 2011.

[10] C Buratti. Performance analysis of IEEE 802.15.4 beacon-enabled mode. IEEE Trans. 2010; 59: 2031-2045.

[11] Z Tao, S Panwar, D Gu, J Zhang. Performance analysis and a proposed improvement for the IEEE 802.15.4 contention access period. IEEE Wireless Communications and Networking Conference. 2006; 4: 1811-1818.

[12] P Park, P Di Marco, P Soldati, C Fischione, KH Johansson. A generalized Markov chain model for effective analysis of slotted IEEE 802.15.4. IEEE $6^{\text {th }}$ International Conference on Mobile Adhoc and Sensor Systems. 2009: 130-139.

[13] YK Huang, AC Pang, HN Hung. A comprehensive analysis of low-power operation for beaconenabled IEEE 802.15.4 wireless networks. IEEE Transactions on Wireless Communications. 2009; 8(11): 5601-5611.

[14] M Khanafer, M Guennoun, HT Mouftah. Adaptive sleeping periods in IEEE 802.15 .4 for efficient energy savings: markov-based theoretical analysis. IEEE International Conference on Communications (ICC), Kyoto, Japan. 2011.

[15] B Gao, C He, L Jiang. Modeling and analysis of IEEE 802.15.4 CSMA/CA with sleep mode enabled. International Conference on Communication Systems. Guangzhou, China. 2008: 6-11.

[16] CY Jung, HY Hwang, DK Sung, GU Hwang. Enhanced Markov Chain Model and Throughput Analysis of the Slotted CSMA/CA for IEEE 802.15.4 under Unsaturated Traffic Conditions. IEEE Transactions on Vehicular Technology. 2009; 58(1): 473-478.

[17] B Shrestha, E Hossain, S Camorlinga. A Markov model for IEEE 802.15.4 MAC with GTS transmissions and heterogeneous traffic in non-saturation mode. IEEE International Conference on Communication Systems (ICCS), Singapore. 2010: 56-61.

[18] BH. Lee, M Udin Harun Al Rasyid, HK. Wu. Analysis of superframe adjustment and beacon transmission for IEEE 802.15.4 cluster tree networks. EURASIP Journal on Wireless Communication and Networking. 2012. 
[19] M Martalo, S Busanelli, G Ferrari. Markov Chain-based performance analysis of multihop IEEE 802.15.4 wireless networks. Performance Evaluation Journal. 2009; 66: 722-741.

[20] HK Wu, BH Lee, M Udin Harun Al Rasyid. Study on Superframe Adjustment for Cluster Tree in Wireless Sensor Networks. Proceedings of the 2012 IEEE International Conference on Cyber Technology in Automation, Control and Intelligent Systems. 2012: 43-47.

[21] BH Lee, Eppy Yundra, HK Wu, M Udin Harun Al Rasyid. Analysis of superframe duration adjustment scheme for IEEE 802.15.4 networks. EURASIP Journal on Wireless Communication and Networking. 2015.

[22] K Sarvakar, PS Patel. An Efficient Hybrid MAC Layer Protocol Utilized for Wireless Sensor Networks. In Proceedings of International Conference on Wireless Communication and Sensor Networks (WCSN), Allahabad, India. 2008: 22-26.

[23] H Deng, J Shen, B Zhang, J Zheng, J Ma, H Liu, Performance Analysis for Optimal Hybrid Medium Access Control in Wireless Sensor Networks. In Proceedings of IEEE Global Telecommunications (GLOBECOM) Conference, New Orleans, LA, USA. 2008: 203-207.

[24] I Rhee, A Warrier, M Aia, J Min, ML Sichitiu. Z-MAC: A Hybrid MAC for Wireless Sensor Networks. IEEE/ACM Trans. Network. 2008; 16; 511-524.

[25] RK Patro, M raina, V Ganapathy, M Shamaiah, C Thejaswi. Analysis and Improvement of Contention Access Protocol in IEEE 802.15.4 star network. IEEE International Conference on Mobile Adhoc and Sensor Systems, MASS 2007. 2007: 1-8.

[26] MB Rasheed, N Javaid, A Haider, U Qasim, ZA Khan, TA Alghamdi. An Energy Consumption Analysis of Beacon Enabled Slotted CSMA/CA IEEE 802.15.4. 28th International Conference on Advanced Information Networking and Applications Workshops (WAINA). 2014: 372-377.

[27] BH Lee, RL Lai, HK Wu, CM Wong. Study on Additional Carrier Sensing for IEEE 802.15.4 Wireless Sensor Networks. Sensors. 2010: 6275-6289.

[28] Ji Z. Design of an integrated controller based on ZigBee wireless sensor network. TELKOMNIKA Indonesian Journal of Electrical Engineering. 2013; 11(8): 4414-4421.

[29] Lu ZQ, Wang LG, Shan J. Research on an Improved Wireless Sensor Networks Clustering Protocol. TELKOMNIKA Indonesian Journal of Electrical Engineering. 2013; 11(10): 5980-5.

[30] B Bougard, F Catthoor, DC Daly, A Chandrakasan, W Dehaene. Energy Efficiency of the IEEE 802.15.4 Standard in Dense Wireless Microsensor Networks: Modeling and Improvement Perspectives. Proceeding of Design, Automation and Test in Europe Conference and Exhibition. (DATE'05). 2005: 196-201.

[31] CM Wong, BH Lee. An Improvement of Slotted CSMA/CA Algorithm in IEEE 802.15.4 Medium Access Layer. Wireless Pers Commun. 2012: 807-822.

[32] AS Chipcon SmartRF® CC2420 datasheet (rev 1.2), Chipcon corp. 2004. 